\section{Infection by Mycobacterium caprae in three cattle herds in Emilia-Romagna Region, Northern Italy}

\author{
Rossella Magnani, ${ }^{1}$ Mauro Cavalca, ${ }^{1}$ \\ Marco Pierantoni, ${ }^{1}$ Andrea Luppi, ${ }^{2}$ \\ Anna Maria Cantoni, ${ }^{3}$ Alice Prosperi, ${ }^{2}$ \\ Maria Pacciarini, ${ }^{2}$ Mariagrazia Zanoni, ${ }^{2}$ \\ Marco Tamba, ${ }^{2}$ Annalisa Santi, ${ }^{2}$ \\ Silvia Bonardi ${ }^{3}$ \\ ${ }^{1}$ National Health Service, Veterinary \\ Service, Local Unit of Parma; ${ }^{2}$ Istituto \\ Zooprofilattico Sperimentale della \\ Lombardia e dell'Emilia-Romagna \\ (IZSLER), Brescia; ${ }^{3}$ Department of \\ Veterinary Science, University of Parma, \\ Italy
}

\begin{abstract}
Bovine tuberculosis (bTB) is a contagious chronic disease associated with progressive emaciation (starvation) and tubercles (granuloma) formation commonly caused by Mycobacterium bovis. In cattle, $M$. caprae may also be responsible for bTB. In EU, human tuberculosis due to $M$. bovis had a notification rate of 0.04 cases per 100,000 inhabitants in 2017 , but data did not include $M$. caprae infections. From September 2018 to April 2019, bTB outbreaks were investigated in three neighbouring dairy cattle herds in Parma province, Northern Italy. Parma municipality belongs to an officially free of bovine tuberculosis (OTF) Italian region. Official testing on cattle herds, performed every three years as legally required, revealed no positive animals. Tubercular lesions were found during the post mortem (PM) examination of slaughtered cattle and $M$. caprae genotype SB0418/VNTR 4,3,5,3,4,5,2,2,4, $3,15,5$ was isolated. This report confirms the crucial importance of PM veterinary inspection at slaughterhouse, despite the OTF status of cattle herds.
\end{abstract}

\section{Introduction}

In EU, human tuberculosis due to Mycobacterium bovis is rare, with 185 cases reported in 2017 and a notification rate of 0.04 cases per 100,000 inhabitants. The notification rate is higher in Member States (MS) not officially free from bovine tuberculosis (non-OTF) compared to MS that were OTF in cattle (EFSA and ECDC,
2018). In a low proportion of human cases of tuberculosis (TB) the causative zoonotic agent is Mycobacterium caprae rather than M. bovis (Prodinger et al., 2014) but EU statistics for TB do not include data of infection due to M. caprae. On the contrary, in cattle, all cases caused by a pathogenic member of the Mycobacterium tuberculosis complex, including $M$. caprae, are included in the statistics provided by the MS (EFSA and ECDC, 2018).

Bovine tuberculosis (bTB) is a contagious chronic disease associated with progressive emaciation and tubercle (granuloma) formation involving usually the respiratory tract but also other organs. Symptoms take usually years to appear and include weakness, loss of appetite, weightloss, fluctuating fever, intermittent hacking cough, diarrhoea, appearance of prominent lymph nodes (OIE, 2019). Beside its economic importance to the livestock industry, its zoonotic nature deserves the greatest attention as public health risk.

$M$. bovis and $M$. caprae are both members of the Mycobacterium tuberculosis complex (MTC), however, while $M$. bovis is acknowledged as associated with bTB, $M$. caprae has been recently classified as a separate species and has been included in the MTC (Perez-Lago et al. 2014).

In Italy, a bTB eradication program is in place since 1995 , but in 2019 only 8 regions and 15 provinces are declared as bTB officially free territories (OTF).

Single intradermal tuberculin skin test (TST) is the official test applied every six months in the herds involved in bTB eradication program. According to the EU regulation, if the bTB herd prevalence decreases below $1 \%$, the herd testing interval could be increased: in Emilia-Romagna, one of the Italian OTF Regions, a three-year testing frequency is performed. The interferongamma blood testing (IFN- $\gamma$ ) is applied as ancillary (laboratory) test for a faster eradication in bTB confirmed infected herds.

\section{Case Report}

\section{Herd 1 (66 cattle)}

On 2018.09.12, tubercular lesions during the post mortem (PM) examination of a slaughtered dairy cow were detected by the official veterinaries. The animal was tested on herd by intradermal tuberculin skin test (TST) on 2016.08.17 and resulted negative, as well as all the other cattle of the herd, tested on 2016.03.03. The PM lesions were represented by the presence of "tubercles" (granulomas) in the tracheobronchial and mediastinal lymph nodes. Lymph nodes at
Correspondence: Silvia Bonardi, Department of Veterinary Science, University of Parma, Strada del Taglio 10, 43126, Parma, Italy. Tel.: +39.0521.032744.

E-mail: silvia.bonardi@unipr.it

Key words: Mycobacterium caprae, dairy cattle, post mortem inspection, Italy.

Conflict of interests: The authors declare no potential conflict of interests.

Contributions: the authors contributed equally. Funding: None.

Availability of data and materials: The data that support the findings of this study are available from the corresponding author upon reasonable request.

Ethics approval and consent to participate: This research was conducted in accordance with all relevant guidelines and procedures.

Consent for publication: The manuscript does not contain any individual person's data in any form.

Received for publication: 3 August 2019.

Revision received: 15 December 2019.

Accepted for publication: 7 January 2020.

This work is licensed under a Creative Commons Attribution-NonCommercial 4.0 International License (CC BY-NC 4.0).

\section{(C) Copyright: the Author(s), 2020}

Licensee PAGEPress, Italy

Italian Journal of Food Safety 2020; 9:8467

doi:10.4081/ijfs.2020.8467

the gross examination appeared enlarged and on cut surface numerous tubercles were recorded; tubercles showed a caseo-necrotic center with a diameter range of 5-10 $\mathrm{mm}$. Pulmonary tubercles were also present. The appearance of the lesions suggested the development of a primary complex. The animal did not show any sign of disease during the ante-mortem visit. Histologically, the tuberculous granulomas in lymph nodes showed Langhans-type multinucleated giant cells, surrounding a caseous necrotic centre. Acid-fast bacilli were observed in the lesions. Samples were submitted to PCR and bacterial isolation according to procedures described by Boniotti et al. (2015). On 2018.11.05 M. caprae was isolated, identified and genotyped by spoligotyping and Multilocus Variable Number Tandem Repeats Analysis (MLVA with 12 loci) according to the methods described by Boniotti et al. $(2009 ; 2015)$. The $M$. caprae isolate belonged to the SB0418/VNTR $4,3,5,3,4,5,2,2,4,3,15,5$ genotype. 
After the first reporting, on 2018.09.25 the local sanitary authorities tested all the cattle by TST, with negative results. On 2018.11.12, TST testing was repeated and two animals reacted positive. PM slaughter examination of the two cows revealed no tubercular lesions and no histological changes in lymph nodes were observed by laboratory testing. On 2018.28.12, TST testing was repeated and all animals on herd reacted negative. On 2019.01.15, the interferon-gamma blood testing (IFN- $\gamma$ ) was performed and 10 cows were positive. All the IFN- $\gamma$ positive animals were slaughtered and four of them showed tubercular lesions in tracheo-bronchial and mediastinal lymph nodes (incomplete primary complex). The lymph nodes of the 10 IFN- $\gamma$-positive animals were sent to the IZSLER laboratories, where 3 samples out of 4 with tubercular lesions showed histopathological changes suggestive of tuberculosis: central caseous necrosis, mineralization foci and Langhans-type multinucleate giant cells. $M$. caprae SB0418/VNTR 4,3,5,3,4, 5,2,2,4,3,15,5 was isolated by culture from the lymph nodes of five animals. Stamping-out of the heard was performed.

\section{Herd 2 (19 cattle)}

In October 2018, following the outbreak confirmation in Herd 1, Herd 2 was subjected to TST test because of epidemiological links with Herd 1 (exchange of equipment and movements of calves). Herd 1 and Herd 2 were separated only by a courtyard. All animals showed negative TST reactions.

On 2018.12.10, tubercular lesions were detected during a dairy cow PM slaughter examination. Tubercles in the retropharyngeal, tracheobronchial and mediastinal lymph nodes (Figure 1) as well as in the pulmonary parenchyma were observed. In addition, nodular and soft lardaceous lesions of $0.5-1 \mathrm{~cm}$ size, resembling pearls were observed on the serosal surfaces, i.e. the pulmonary and parietal pleura and visceral peritoneum (Figure 2) (protracted generalization).

On 2018.12.11, TST testing was repeated and one cow showed an inconclusive reaction. At slaughter, PM examination showed tubercular lesions only in the tracheobronchial lymph nodes (incomplete primary complex). Histopathology revealed lymph nodes lesions suggestive of tuberculosis characterized by granulomas with central caseous necrosis, surrounded by epithelioid macrophages and Langhanstype giant cells. Cultural detection of the microorganism resulted negative due to sample contamination. On 2019.01.29, another cow showed at PM examination multiple tuberculous lesions of $4-5 \mathrm{~mm}$ size in the mediastinal lymph nodes (incomplete primary complex). bTB was confirmed by histological examination of the lymph node, which showed multiple granulomas with central areas of caseous necrosis, a wall of epithelioid macrophages and Langhans-type giant cells. Cultural testing isolated the $M$. caprae SB0418/VNTR 4,3,5,3,4,5,2, 2,4,3,15,5 genotype as in Herd 1. On 2019.01.29, all cattle were investigated by TST and IFN- $\gamma$ testing. One cow positive for TST and 15 cows positive by IFN- $\gamma$ test were found. Positive cattle were slaughtered and tracheobronchial or mediastinal lymph nodes tuberculous lesions referring to an incomplete primary complex were detected in all of them. Stamping-out was performed.

\section{Herd 3 (123 cattle)}

In October 2018, following the outbreak confirmation in Herd 1, TST testing of all cattle was performed, because one animal had been previously moved from Herd 2 to Herd 3. All animals showed a negative TST reaction.

On 2019.02.11, TST testing was repeated in all cattle and two animals resulted positive (in addition, two inconclusive TST reactions were observed). At slaughterhouse, one TST-positive cow showed lesions in tracheobronchial and mediastinal lymph nodes, resembling tubercolosis. Histological samples of granulomatous lesions were characterized by central areas of caseous necrosis and mineralization, epithelioid and Langhans-type giant cells. Lesions were attributed to primary infection (incomplete primary complex). bTB was confirmed by the isolation of $M$. caprae SB0418/VNTR 4,3,5,3,4,5,2, $2,4,3,15,5$ from the lymph nodes.

On 2019.02.26, IFN- $\gamma$ testing on cattle revealed 19 positive cases, as well as 11 inconclusive results. On 2019.04.09, both TST and IFN- $\gamma$ testing were repeated on cattle farm. Two TST-reactors were found, as well as 13 inconclusive TST reactions. In addition, 20 animals were found to be positive to the IFN- $\gamma$ test.

Cattle stamping out was performed. At slaughter, no tuberculous lesions were observed at PM examination in any cattle. Samples of TST and IFN- $\gamma$ positive cattle (retropharyngeal, bronchial, mesenteric and popliteal lymph nodes, together with tonsils) were tested by the IZSLER laboratories. Neither histopathological lesions nor mycobacteria growth in culture were observed.

\section{Discussion}

The accuracy of the TST test varies widely due to different factors, based on the host and the test procedure itself. The exact estimation of the TST test performance in the field is therefore difficult (Monaghan et al., 1994; de la Rua-Domenech et al., 2006; Alvarez et al, 2012). However, intradermal skin tests normally have a high specificity, although sensitivity can be somewhat lower (de la Rua-Domenech et al., 2006). The use of IFN- $\gamma$ test in parallel to the TST can implement the number of infected animals detected in the target population. This strategic use of the IFN- $\gamma$ test increases the

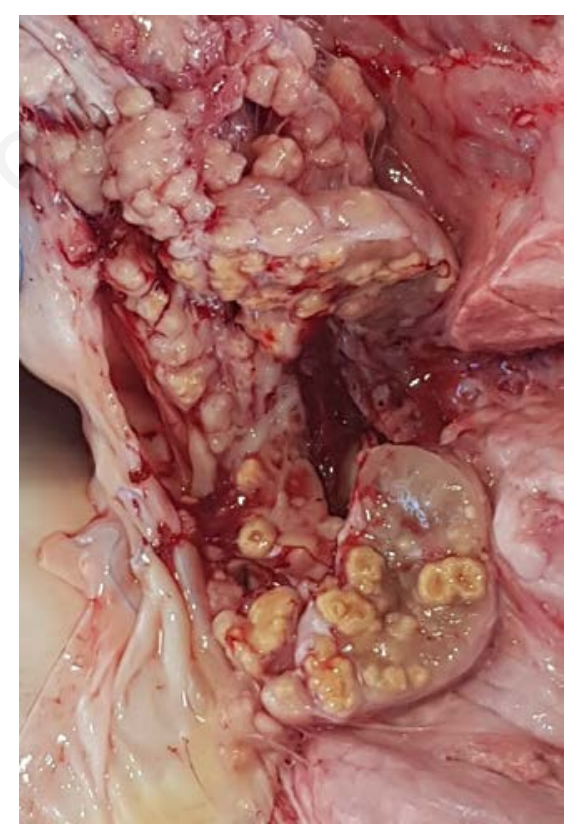

Figure 1. Tubercles in mediastinal lymph nodes (Herd 2; PM inspection on 2018.12.10).

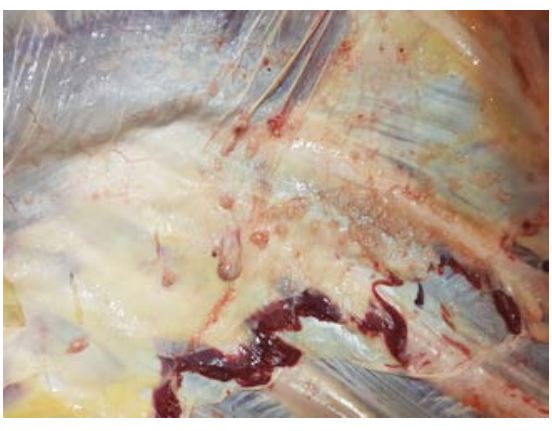

Figure 2. Tubercles in serosal surface (pleura) (Herd 2; PM inspection on 2018.12.10). 
sensitivity of the diagnostic procedure,but decreases its specificity (EFSA 2012; OIE 2019). However, cleaning and disinfection of the premises, after depopulation of the whole herd, seem to be the faster methods to maintain cattle population free from bTB infection in case of an outbreak.

Epidemiological links among the outbreaks were found. In particular, three calves were moved from Herd 2 to Herd 1 between late October 2016 and January 2017; interestingly, one of these calves was born from the cow belonging to Herd 2 which showed tubercular lesions at slaughter on 2018.12.10. In addition, a heifer was moved from Herd 1 to Herd 3. As a consequence, Herd 2 was identified as the primary outbreak.

M. caprae genotype SB0418/VNTR $4,3,5,3,4,5,2,2,4,3,15,5$ was isolated from cattle reared in all the herds. The same strain was first isolated from cattle reared in Trento province in 2006 and in EmiliaRomagna Region (Reggio Emilia Province) in 2011, as well as in other Regions of Northern Italy (Lombardy and Veneto) (Pacciarini, personal communication). These findings suggest that the same mycobacterial strain is still circulating among herds of OTF Regions of Italy.

Following Commission Delegated Regulation (EU) 2019/627 concerning specific rules for the performance of official controls on the production of meat, PM inspection is performed by the Official Veterinarian in case of cattle coming from herds which are not OTF. On the contrary, for animals coming from OTF herds on the basis of TST testing, as was the case described in the present study, PM inspection could be performed by an Official Auxiliary under the responsibility of the Official Veterinarian, referred to Article 18 (2) (c) of Regulation (EU) 2017/625. Nevertheless, this bTB case underlines that, despite the OTF status of a herd, PM inspection of cattle at slaughter is crucial to monitor the appearance of cases of bTB in a territory and protect human health.

\section{Conclusions}

Despite the application of all measures required for eradication of bTB, Italy is not an OTF country. As observed in the present bTB outbreaks, the appearance of gross tuberculous lesions at slaughter was detected two years and a half after the herd TST. Nevertheless, infected cattle frequently gave negative results at TST.

Movement of animals is the main risk factor for a new introduction of bTB in an OTF herd. Pre- or post-movement testing do not completely eliminate the risk of transmission of mycobacteria among herds, even in OTF areas, due to the low sensitivity of TST.

In addition to the discussion on sensitivity and specificity of the diagnostic tests used on live animals, this paper highlights the importance of Official Veterinary post mortem inspection at slaughterhouse. PM examination of the lymph nodes of the head and respiratory tract, together with examination of lungs and serosal surfaces, was essential for the detection of suspected bTB lesions. Therefore, veterinary inspection at slaughter, combining ante- and post mortem examinations of animals and their carcasses, should still be considered "the gold standard" to ensure meat safety and to protect human and animal health.

\section{References}

Alvarez J, Perez A, Bezos J, Marqués S, Grau A, Saez JL, Mínguez O, de Juan L, Domínguez L, 2012. Evaluation of the sensitivity and specificity of bovine tuberculosis diagnostic tests in naturally infected cattle herds using a Bayesian approach. Vet Microbiol 155:38-43.

Boniotti MB, Goria M, Loda D, Garrone A, Benedetto A, Mondo A, Tisato E, Zanoni M, Zoppi S, Dondo A, Tagliabue S, Bonora S, Zanardi G, Pacciarini ML, 2009. Molecular typing of Mycobacterium bovis strains isolated in Italy from 2000 to 2006 and evaluation of Variable-NumberTandem-Repeats for a geographic optimized genotyping. J Clin Microbiol 47:636-44.
Boniotti MB, Gaffuri A, Gelmetti D, Tagliabue S, Chiari M, Mangeli A, Spisani M, Nassuato C, Gibelli L, Sacchi C, Zanoni M, Pacciarini ML, 2014. Detection and molecular characterization of Mycobacterium microti in wild boar from Northern Italy. J Clin Microbiol 52:2834-43.

de la Rua-Domenech R, Goodchild AT, Vordermeier HM, Hewinson RG, Christiansen KH, Clifton-Hadley RS, 2006. Ante mortem diagnosis of tuberculosis in cattle: a review of the tuberculin tests, gamma-interferon assay and other ancillary diagnostic techniques. Res Vet Sci 81:190-210.

Domingo M, Vidal E, Marco A, 2014. Pathology of bovine tuberculosis. Research Vet Sci 97:S20-29.

European Food Safety Authority (EFSA), 2012. Scientific Opinion on the use of a gamma interferon test for the diagnosis of bovine tuberculosis. EFSA J 10:2975.

European Food Safety Authority (EFSA), European Centre for Disease Prevention and Control (ECDC), 2018. The European Union summary report on trends and sources of zoonoses, zoonotic agents and food-borne outbreaks in 2017. EFSA J 16:5500.

Monaghan ML, Doherty ML, Collins JD, Kazda JF, Quinn PJ, 1994. The tuberculin test. Vet Microbiol 40:111-24.

OIE (World Organization for Animal Health), 2009. Manual of diagnostic tests and vaccines for terrestrial animals. Bovine tuberculosis. Chapter 3.4.6. pp. 1058-74.

OIE (World Organization for Animal Health). Bovine tuberculosis (accessed 06.07.2019)

Perez-Lago L, Navarro Y, Garcìa-deViedma D, 2014. Current knowledge and pending challenges in zoonosis caused by Mycobacterium bovis: a review. Res Vet Sci 97:S94-S100.

Prodinger WM, Indra A, Koksalan OK, Kilikaslan Z, Richter E, 2014. Mycobacterium caprae infection in humans. Expert Rev Anti Infect Ther 12:1501-13. 